\title{
Mathematisches Praktikum für Schüler
}

\author{
von Aloys Krieg
}

Vor dem Hintergrund sinkender Studentenzahlen gibt es an vielen Universitäten Überlegungen, wie man diesem Trend entgegenwirken und wieder mehr Abiturienten für das Mathematikstudium gewinnen kann. An der RWTH Aachen wurde zu Beginn des Sommersemesters eine neue Initiative gestartet, in der die Fachgruppe Mathematik Praktikumsplätze für Schüler anbietet.

An vielen Gymnasien wird zu Beginn der Oberstufe ein zweiwöchiges Betriebspraktikum durchgeführt. Dies ist ein besonders günstiger Zeitpunkt, die weitere Entwicklung der Schüler noch zu beinflussen, weil die Wahl der Leistungskurse erst am Ende der 11. Klasse getroffen wird. Das Angebot für Praktikumsplätze wurde an die Schulen der Aachener Region weitergegeben. Die Reaktion ist positiv, weil sich inzwischen mehr als 20 Schülerinnen und Schüler angemeldet haben. Darüber hinaus wurde auch Interesse bekundet, sich z. B. in den Oster- oder Sommerferien im Rahmen eines solchen Praktikums über das Mathematikstudium zu informieren. Bei dieser Aktion wurde wiederum deutlich, wie wichtig die Rolle der Lehrerschaft als Multiplikator ist. Das größte Interesse kam von den Schulen, an denen die Lehrerinnen und Lehrer dieses Thema alktiv aufgegriffen, gefördert und potentielle Interessenten direkt angesprochen haben.

Was macht man aber mit Schülern, die weder differenzieren noch integrieren können, sondern gerade erst die Mittelstufe absolviert haben? Der Grundgedanke des Praktikums besteht darin, die Freude an der Mathematik zu wecken bzw. zu fördern und den entdeckenden Charakter der Mathematik, der im Unterricht aufgrund der engen Vorgaben fast zwangsläufig zu kurz kommt, zum Vorschein zu bringen.

Zunächst einmal erhalten die Schülerinnen und Schüler eine Einführung in MAPLE. Dr. Ulrich Klein vom Lehrstuhl D für Mathematik, RWTH Aachen, ist in diesem Praktikum mit großem persönlichen Engagement federführend tätig. Er vermittelt die Einführung und hat eine ganze Reihe von Arbeitsblättern vorbereitet, die zum Schulstoff der Mittel- stufe passen. Ein wesentlicher Aspekt ist hier die praktische Anwendung von MAPLE zum Beispiel auf lineare Gleichungssysteme.

Als weiteres Arbeitsgebiet wird die Geometrie vorgestellt. Zunächst wird erklärt und geübt, wie man mit verschiedenen Zeichenprogrammen auf dem Computer z. B. Dreiecke konstruieren kann. Mit „angeleitetem" Entdecken werden die Schülerinnen und Schüler dann auf die Euler-Gerade oder den Satz von Feuerbach geführt, die sicherlich in der Schule nicht behandelt werden. Hierzu wird der dynamische Aspekt der Computerprogramme ausgenutzt, weil man sofort sieht, wie sich Höhenschnittpunkt und Schwerpunkt verändern, wenn man zum Beispiel einen Eckpunkt verschiebt.

Andere Arbeitsgebiete, die in Aachen vorgestellt werden, sind z.B. Graphentheorie, Wahrscheinlichkeitstheorie und Statistik sowie die numerische Mathematik. Vertreter dieser Gebiete gestalten jeweils ein, zwei oder drei Tage des Praktikums, so dass sich die Belastung für jeden einzelnen Lehrstuhl in Grenzen hält.

Dies ist sicherlich nur eine Maßnahme unter vielen, um das Interesse an einem Mathematikstudium zu fördern. Die Resonanz zeigt aber deutlich, wie wichtig es für die Universitäten ist, auf die Schulen zuzugehen und den Dialog und die Kooperation zu suchen.

\section{Adresse des Autors}

Prof. Dr. Aloys Krieg

Lehrstuhl A für Mathematik

Rheinisch-Westfälische Technische Hochschule

52056 Aachen

krieg@mathA.rwth-aachen.de 